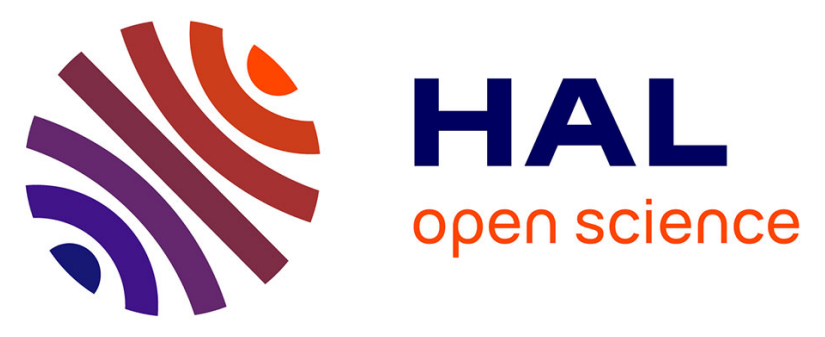

\title{
On the stability of Be3: A benchmark complete active space self-consistent field + averaged quadratic coupled cluster study
}

J. I. Amaro-Estrada, Anthony Scemama, Michel Caffarel, Alejandro

Ramirez-Solis

\section{To cite this version:}

J. I. Amaro-Estrada, Anthony Scemama, Michel Caffarel, Alejandro Ramirez-Solis. On the stability of Be3: A benchmark complete active space self-consistent field + averaged quadratic coupled cluster study. Journal of Chemical Physics, 2011, 135 (10), pp.104311/1-104311/7. 10.1063/1.3635403 . hal-00875418

\section{HAL Id: hal-00875418 https://hal.science/hal-00875418}

Submitted on 29 Jan 2020

HAL is a multi-disciplinary open access archive for the deposit and dissemination of scientific research documents, whether they are published or not. The documents may come from teaching and research institutions in France or abroad, or from public or private research centers.
L'archive ouverte pluridisciplinaire HAL, est destinée au dépôt et à la diffusion de documents scientifiques de niveau recherche, publiés ou non, émanant des établissements d'enseignement et de recherche français ou étrangers, des laboratoires publics ou privés.

$$
\text { Copyright }
$$




\section{AIP}

On the stability of Be3: A benchmark complete active space self-consistent field + averaged quadratic coupled cluster study

J. I. Amaro-Estrada, A. Scemama, M. Caffarel, and A. Ramírez-Solís

Citation: J. Chem. Phys. 135, 104311 (2011); doi: 10.1063/1.3635403

View online: http://dx.doi.org/10.1063/1.3635403

View Table of Contents: http://jcp.aip.org/resource/1/JCPSA6/v135/i10

Published by the American Institute of Physics.

\section{Additional information on J. Chem. Phys.}

Journal Homepage: http://jcp.aip.org/

Journal Information: http://jcp.aip.org/about/about_the_journal

Top downloads: http://jcp.aip.org/features/most_downloaded

Information for Authors: http://jcp.aip.org/authors 


\title{
On the stability of $\mathrm{Be}_{3}$ : $\mathrm{A}$ benchmark complete active space self-consistent field + averaged quadratic coupled cluster study
}

\author{
J. I. Amaro-Estrada, ${ }^{1}$ A. Scemama, ${ }^{2}$ M. Caffarel, ${ }^{2}$ and A. Ramírez-Solís ${ }^{3, a)}$ \\ ${ }^{1}$ Instituto de Ciencias Físicas, Universidad Nacional Autónoma de México, A.P. 48-3, Cuernavaca, Morelos \\ 62251, México \\ ${ }^{2}$ CNRS-Laboratoire de Chimie et Physique Quantiques, IRSAMC, Université de Toulouse, France \\ ${ }^{3}$ Departamento de Física, Facultad de Ciencias, Universidad Autónoma del Estado de Morelos, Av. \\ Universidad 1001. Cuernavaca, Morelos. 62209, México
}

(Received 8 June 2011; accepted 18 August 2011; published online 12 September 2011)

\begin{abstract}
The optimized geometries and binding energies for the linear and triangular isomers of the beryllium trimer have been obtained through benchmark multireference averaged quadratic coupled cluster (AQCC) calculations using very large complete active space SCF (CASSCF) references (12 active electrons in 13 and 14 orbitals). Geometries were optimized with the cc-pV5Z basis, while the binding energies (including counterpoise correction) were obtained with the significantly larger aug-ccpV5Z basis set. The binding energies $(27.3$ and $16.3 \mathrm{kcal} / \mathrm{mol}$ for the equilateral and linear isomers, respectively) are larger than the previous full $\mathrm{CI}$ benchmark values, while the corresponding Be-Be equilibrium distances of 4.101 and 4.088 a.u. are smaller. In view of the near-size consistency character of the CASSCF + AQCC method, the fact that all 12 electrons are fully correlated, the active reference space includes 14 orbitals, and the very large basis set used here, we propose to consider these results as reference data for $\mathrm{Be}_{3}$. Using the electron pair localization function obtained at the CASSCF $(12,15)$ level, it is clearly illustrated that the $2 p$ orbitals lying in the molecular plane play a dominant role in the bonding pattern for the equilateral isomer. () 2011 American Institute of Physics. [doi:10.1063/1.3635403]
\end{abstract}

\section{INTRODUCTION}

The structure and binding energies of the beryllium dimer and trimer have been widely studied, ${ }^{1-36}$ both theoretically and experimentally. The dimer is experimentally known to be very weakly bound and, since it contains only eight electrons, the accurate theoretical description of its ground (singlet) state potential surface has been the subject of many works since the early days of quantum chemistry. A large number of studies have shown that the bonding in the dimer is actually due to contributions of the hybridization of the $2 s$ and $2 p$ orbitals, and the non-dynamic correlation effects that arise are, therefore, crucial to properly define zeroth-order electronic wavefunctions. Many electronic structure methods ranging in complexity (perturbational up to fourth order, singleand multi-reference variational CI, density functional theory (DFT)-based, coupled cluster, and even full CI (FCI)) have been applied to study $\mathrm{Be}_{2}$ (Refs. 3-5, 8-10, 17, 18, 21, 24-26) and $\mathrm{Be}_{3}$ (Refs. 4, 5, 8, 10-13, 15, 20-24, 27, 28) using, also, many types of atomic basis sets. In the case of the Be dimer, a number of quantum Monte Carlo (QMC) approaches have also been used (see Refs. 30-36). Since Be exists in the bulk as a metallic solid, it is natural to raise the question of the evolution of the binding energy (BE) per atom as the number of atoms grows. For larger Be clusters, it has been found that, in spite of the fact that no $d$ orbitals are involved in the bond shells, as mentioned by Junquera et al. ${ }^{23}$ the presence of the unoccupied $2 p$ orbitals, which are quasi-degenerate with

\footnotetext{
a)Electronic mail: alex@uaem.mx.
}

the $2 s$ orbitals, allows the formation of Be clusters by this $s p 2$ hybridization process. Actually, it has been shown that this hybridization grows with the number of Be atoms in the cluster. ${ }^{5}$ Kaplan et al. ${ }^{21}$ have argued that the binding in $\mathrm{Be}_{3}$ has a mixed physical (van der Waals) and chemical (nonadditive exchange) nature, while the $2 s$ - $2 p$ quasi-degeneracy leads to strong multiconfigurational character in the description of the wavefunctions for both dimer and trimer (see, for instance, Refs. 8, 12, 13, 18, 19, 24). Lee et al. ${ }^{11}$ made a careful comparison of the electronic description at the MRCI and CCSD levels for $\mathrm{Be}_{n}(n=3-5)$ clusters. They concluded that the coupled cluster approach restricted to single and double excitations is not capable of quantitative accuracy in the description of the electronic structure for Be clusters, since this method underestimates the dissociation energies.

For the $\mathrm{Be}_{2}$ van der Waals dimmer, it is well known, both theoretically ${ }^{3-5,8-10,16-18,21,24-26,30-36}$ and experimentally, ${ }^{6,7,14,19,29}$ that the interaction energy is quite small, around $2.4 \mathrm{kcal} / \mathrm{mol}$, while the Be-Be distance is rather large, around $2.4 \AA$. This rather small binding energy for the dimer is in contrast with the non-negligible binding energy per bond (around $13 \mathrm{kcal} / \mathrm{mol}$ ) for the bulk. ${ }^{11}$ So, it has been proposed that the existence of $\mathrm{Be}$ in the bulk can be explained through an increase of the $\mathrm{BE}$ for larger $\mathrm{Be}_{n}$ clusters. Based on the previous idea, density functional techniques were applied to address beryllium clusters $\mathrm{Be}_{n}(n=2-8)$ by Beyer et al. ${ }^{20}$ using a large $6-311++\mathrm{G}(3 d f)$ basis set to study the structural and energetic properties as functions of cluster size and to determine their convergence towards metallic beryllium. 
Unlike $\mathrm{Be}_{2}$, to the best of our knowledge, for the trimer there are no experimental values to compare with. However, there is a large number of theoretical works using many types of electronic structure methods. ${ }^{4,5,8,10-13,15,20-24,27,28}$ All these studies highlight the importance of an accurate treatment of both dynamic and non-dynamic correlation effects, coupled to the use of extended atomic basis sets including higher l-angular momentum orbitals. For $\mathrm{Be}_{3}$, both the geometry and the binding energy are known to be strongly dependent on such factors. Here, our goal is to present the results of new benchmark-type multireference variational calculations for the linear and triangular isomers of $\mathrm{Be}_{3}$ using the largest augmented correlation-consistent Gaussian atomic basis sets defined for the beryllium atom. Section II deals with the theoretical method and the computational details, including the treatment of the basis set superposition error (BSSE) which is known to be important for the binding energy. In the same section, we recall the fundamentals of the electron pair localization function (EPLF) we will be using to study the binding pattern of the equilateral isomer. In Sec. III, we present the structural and energetic results compared with previous values. Finally, Sec. IV presents the conclusions and some perspectives.

\section{METHOD AND COMPUTATIONAL DETAILS}

\section{A. Basis sets and non-dynamic/dynamic correlation treatments}

Since we are interested in improving the most accurate values of the binding energy presently available for the Be trimer, some important aspects must be taken into account. First, in view of the strong dependence of the binding energy on the largest l-angular momentum atomic functions used, the highest-quality available basis sets have to be chosen. Thus, we have employed two of the most sophisticated atomic Gaussian basis sets existing for this atom, namely, the cc-pV5Z and aug-cc-pV5Z (AV5Z) basis sets of Dunning et al. ${ }^{37,38}$ The latter correlation-consistent $15 s 9 p 5 d 4 f 3 g 2 h$ basis set is contracted to [7s6p5d $f f 3 g 2 h]$ and leads here to a molecular basis of 381 orbitals for 12 electrons in $\mathrm{Be}_{3}$. Second, as stressed by many authors, both non-dynamic and dynamic correlation effects are crucial to properly describe the atomic interactions in $\mathrm{Be}_{3}$ (see, for instance, Refs. 8, 11, 12, 23, 24, 27, 28). In particular, the $\mathrm{Be}-\mathrm{Be}$ bonds in $\mathrm{Be}_{3}$ must be described using a zeroth-order wavefunction that includes, at least, the $2 p$ orbitals that lie in the molecular plane. It is well known that the core-valence correlation effects are crucial to study this system; $8,17,24,27,28$ therefore, we have used the complete active space self-consistent field (CASSCF) approach to build zeroth-order wavefunctions considering four active electrons per atom, thus including all 12 active electrons of the trimers. Since the $2 p$ shells are nearly degenerate with the $2 s$ one, the ideal chemically relevant active space would consist of five orbitals per atom $\left(1 s, 2 s, 2 p_{\mathrm{x}}, 2 p_{\mathrm{y}}\right.$, and $\left.2 p_{\mathrm{z}}\right)$, thus leading to reference wavefunctions built from $\operatorname{CASSCF}(12,15)$ calculations. However, with the present basis set, this ideal active orbital space yields zeroth-order wavefunctions containing more than $1.6 \times 10^{6}$ configuration state functions (CSFs) in the $\mathrm{D}_{3 \mathrm{~h}}$ case; this reference space leads to computationally intractable MRCI calculations involving more than $9 \times 10^{10}$ uncontracted CSFs. Therefore, we have used a two-step procedure that will be explained below. The CASSCF wavefunctions were used as references to perform averaged quadratic coupled cluster (AQCC) (Ref. 39) calculations which, by construction, yield size-consistent energies. Here, it is crucial to highlight that this method has been shown to yield potential energy surfaces parallel to FCI ones with a much reduced computational cost. ${ }^{17}$

\section{B. Geometry optimizations at the CASSCF $(12,12)+$ AQCC level}

In order to perform the geometry optimizations, since the Be-Be bonding occurs mainly in the plane defined by the three atoms (here chosen as the xy plane), a reasonable choice is to exclude the three $2 p_{\mathrm{z}}$ orbitals from the active space, thus obtaining $\operatorname{CASSCF}(12,12)$ references for both trimers. For the triangular molecule, the geometry optimizations have been performed under the $C_{2 v}$ point group (which includes the more symmetric $\mathrm{D}_{3 \mathrm{~h}}$ group). The optimizations of the linear cluster $\left(D_{\infty h}\right.$ structure $)$ have been performed in the $D_{2 h}$ finite group. The geometries of both trimers were optimized using the automatic numerical algorithm at the AQCC level using the CASSCF $(12,12)$ references and the cc-pV5Z basis set $^{44}$ with the MOLPRO-2006.1 code. ${ }^{40}$ Note that even with this basis set, which yields 273 molecular orbitals, the AQCC calculations include 4024119522 (27 890603 ) uncontracted (contracted) CSFs. Therefore, using as starting point, the optimized geometries reported by Junquera $e t$ al. ${ }^{23}$ for the triangular cluster and from Vetere $e t a l .{ }^{24}$ for the linear isomer, the CASSCF $(12,12)+$ AQCC/cc-pV5Z optimizations required each more than a week of CPU time allocating up to $18 \mathrm{~GB}$ of RAM.

\section{CASSCF + AQCC/aug-cc-pV5Z calculations}

Once the optimal geometries were determined at the $\operatorname{CASSCF}(12,12)+$ averaged coupled pair functional (ACPF)/cc-pV5Z level, we proceeded to perform single point calculations using the larger aug-cc-pV5Z basis set considering the largest possible active reference space that our computational resources allowed, i.e., 12 electrons in 13 and 14 active MOs. In the latter case, this means that only one of the molecular orbitals involving the $\left\{2 p_{\mathrm{z}}(1), 2 p_{\mathrm{z}}(2), 2 p_{\mathrm{z}}(3)\right\}$ set of AOs was excluded from the optimal reference space, thus having the lowest natural occupation number in the $\operatorname{CASSCF}(12,15)$ calculation. Although there are several variational CI-based studies for $\mathrm{Be}_{3},{ }^{8,23,24,27}$ we note that the largest FCI calculations done to date are those reported in 2009 by Vetere et al., ${ }^{24}$ where the dimension of their CI spaces was 344545308 Slater determinants with the $5 s 3 p 2 d$ atomic natural orbital (ANO) basis set (with 72 molecular orbitals for $\mathrm{Be}_{3}$ ), but these authors kept the six $1 s$ core electrons frozen and only studied the linear trimer. The final variational space of the present AQCC/AV5Z calculation using the CASSCF $(12,14)$ reference includes 46667364646 (240 820 790) uncontracted (contracted) CSFs for the $\mathrm{D}_{3 \mathrm{~h}}$ 
TABLE I. Details of the CASSCF and ACPF calculations for the ${ }^{1} \mathrm{~A}_{1}$ state of triangular $\mathrm{Be}_{3}$ with the aug-cc-pV5Z basis set. The numbers in parentheses correspond to the $A_{1}, B_{1}, B_{2}$, and $A_{2}$ irreps of the $C_{2 v}$ group, respectively.

\begin{tabular}{lll}
\hline \hline & $\operatorname{CASSCF}(12,13)$ & $\operatorname{CASSCF}(12,14)$ \\
\hline Active orbitals & $13(7510)$ & $14(7520)$ \\
Slater determinants & 740720 & 2262121 \\
CSFs in reference & 198598 & 545857 \\
Averaged quadratic coupled cluster calculations with CASSCF references \\
Internal configs & 198598 & 545857 \\
Contracted configs & 94763087 & 240820790 \\
Uncontracted configs & 19875254627 & 46667364646 \\
\hline \hline
\end{tabular}

cluster. For the linear isomer, the $\operatorname{CASSCF}(12,14)$ includes 277697 CSFs, which generate variational AQCC spaces with 23268073773 (120696813) uncontracted (contracted) CSFs. Note that CI energy convergence to $10^{-6}$ a.u. took $10 \mathrm{CPU}$ days allocating $32 \mathrm{~GB}$ of RAM for the $\mathrm{D}_{3 \mathrm{~h}}$ isomer. Table I presents the details of the CASSCF and AQCC variational spaces for this isomer.

As has been noted before, both the dimer ${ }^{17}$ and the trimer, ${ }^{8,23,24,27}$ are strongly multiconfigurational due to the fact that the nearly degenerate $2 p$ orbitals play an important role in the bonding pattern of these species. As an example of this, Table II presents the composition of the optimized CASSCF $(12,15) / A V 5 Z$ wavefunction of the $\mathrm{D}_{3 \mathrm{~h}}$ trimer, where a rather large number of CSFs appear with CI coefficients larger than 0.05 in absolute value; in particular, note the very low $0.86 * 0.86=0.79$ weight of the HF configuration.

TABLE II. List of CSF in second quantized notation with CI coefficients larger than 0.05 in the $\operatorname{CASSCF}(12,15)$ wavefunction of $\operatorname{Be}_{3}\left(D_{3 h}\right)$ at the equilibrium geometry.

\begin{tabular}{ccccr}
\hline \hline & \multicolumn{2}{c}{$\mathrm{C}_{2 \mathrm{v}}$ Irreps } & & \\
\hline $\mathrm{A}_{1}$ & $\mathrm{~B}_{1}$ & $\mathrm{~B}_{2}$ & $\mathrm{~A}_{2}$ & CI coefficient \\
\hline 2222000 & 22000 & 000 & 0 & 0.8650686 \\
2222200 & 20000 & 000 & 0 & -0.1771835 \\
2220020 & 22000 & 000 & 0 & -0.1265278 \\
2222000 & 20200 & 000 & 0 & -0.1015432 \\
2220000 & 22200 & 000 & 0 & -0.0951988 \\
2220200 & 22000 & 000 & 0 & -0.0948863 \\
$222 \mathrm{bb} 00$ & $2 \mathrm{aa} 00$ & 000 & 0 & -0.0938290 \\
$222 \mathrm{aa} 00$ & $2 \mathrm{bb} 00$ & 000 & 0 & -0.0938290 \\
$2220 \mathrm{ba} 0$ & 22000 & 000 & 0 & 0.0904947 \\
$2220 \mathrm{ab} 0$ & 22000 & 000 & 0 & -0.0904947 \\
2220000 & 22000 & 200 & 0 & -0.0887784 \\
$2222 \mathrm{ba} 0$ & 20000 & 000 & 0 & -0.0883776 \\
$2222 \mathrm{ab} 0$ & 20000 & 000 & 0 & 0.0883776 \\
$222 \mathrm{ab} 00$ & $2 \mathrm{ba} 00$ & 000 & 0 & 0.0863489 \\
$222 \mathrm{ba} 00$ & $2 \mathrm{ab} 00$ & 000 & 0 & 0.0863489 \\
2222020 & 20000 & 000 & 0 & -0.0762465 \\
2202000 & 22000 & 200 & 0 & -0.0728661 \\
2220220 & 20000 & 000 & 0 & 0.0593958 \\
2220200 & 20200 & 000 & 0 & 0.0568272 \\
$22 \mathrm{ba} 000$ & 22000 & $\mathrm{ab} 0$ & 0 & -0.0552713 \\
$22 \mathrm{ab} 000$ & 22000 & $\mathrm{ba} 0$ & 0 & -0.0552713 \\
$222 \mathrm{a} 0 \mathrm{~b} 0$ & $2 \mathrm{ab} 00$ & 000 & 0 & -0.0545349 \\
$222 \mathrm{~b} 0 \mathrm{a} 0$ & $2 \mathrm{ba} 00$ & 000 & 0 & -0.0545349 \\
\hline \hline & & & & \\
\hline
\end{tabular}

\section{The binding energies and inclusion of the counterpoise correction}

It is well known that in order to obtain accurate binding energies for $\mathrm{Be}_{n}$ clusters, the counterpoise (CP) correction ${ }^{41}$ has to be taken into account, since the basis set superposition error plays a very important role. ${ }^{17}$ Therefore, we shall report $\mathrm{BE}$ obtained with and without the $\mathrm{CP}$ corrections (with respect to $3 \mathrm{Be}$ ) for both trimers. The $\mathrm{CP}$ correction can be automatically calculated using the Molpro algorithm; however, for the present case, there is a technical limitation that arises due to the high symmetry of these $\mathrm{Be}_{3}$ isomers. With the MOLPRO program, it is not possible to make the CP correction for symmetry-equivalent ghost atoms, so we had to reduce the symmetry from $\mathrm{C}_{2 \mathrm{v}}$ to use the $\mathrm{C}_{\mathrm{s}}$ point group by using slightly modified geometries involving changes of $10^{-4}$ a.u. in the optimized x-y coordinates of both isomers. For methodological coherence, the $\mathrm{CP}$-corrected binding energies were calculated using the CASSCF $(4,13 / 14)+$ AQCC CP-corrected atomic reference energies and the energies of the optimized trimers at the CASSCF(12,13/14) + AQCC/AV5Z level, with the usual definition, $\mathrm{BE}\left(\mathrm{D}_{3 \mathrm{~h}}\right)=\mathrm{E}\left(\mathrm{D}_{3 \mathrm{~h}}\right)-3 \mathrm{E}(\mathrm{Be})$ for the equilateral isomer and $\mathrm{BE}($ linear $)=\mathrm{E}($ linear $)-2 \mathrm{E}\left(\mathrm{Be}^{*}\right)-$ $\mathrm{E}\left(\mathrm{Be}^{\mathrm{c}}\right)$, where $\mathrm{Be}^{\mathrm{c}}$ and $\mathrm{Be}^{*}$ are the central and end atoms of the linear isomer, respectively. For instance, the CP-corrected atomic energies are -14.6476853 and -14.6476855 a.u. at the CASSCF $(4,13)+$ AQCC and $\operatorname{CASSCF}(4,14)+$ AQCC levels using the optimized $\mathrm{D}_{3 \mathrm{~h}}$ geometry for the ghosts. For comparison, the atomic CP-uncorrected AQCC/AV5Z energy is -14.646173 a.u. using the $1 s, 2 s, 2 p_{\mathrm{x}}, 2 p_{\mathrm{y}}, 2 p_{\mathrm{z}}$ orbitals as active in the $\operatorname{CASSCF}(4,5)$ atomic reference; this energy was used to obtain the $\mathrm{CP}$-uncorrected $\mathrm{BE}$ for the trimers at the CASSCF $(12,13)+$ AQCC and CASSCF $(12,14)+$ AQCC levels.

\section{E. The electron pair localization function}

Let us briefly present the electron pair localization function. The EPLF is a local scalar function defined in the ordinary 3D space, bounded above and below, which focuses essentially on the localization of electron pairs. It is a good descriptive tool for chemical bonds, since pairs of electrons play a central role in our everyday interpretation of chemical structure and reactivity (Lewis model, VSEPR). The original framework proposed to calculate such a localization function is that of quantum Monte Carlo approaches, ${ }^{42}$

$$
\operatorname{EPLF}(\mathbf{r})=\frac{d_{\sigma \sigma}(\mathbf{r})-d_{\sigma \bar{\sigma}}(\mathbf{r})}{d_{\sigma \sigma}(\mathbf{r})+d_{\sigma \bar{\sigma}}(\mathbf{r})},
$$

where

$$
d_{\sigma \sigma}(\mathbf{r})=\left\langle\psi\left|\sum_{i=1}^{N} \delta\left(\mathbf{r}-\mathbf{r}_{i}\right) \min _{j \neq i ; \sigma_{i}=\sigma_{j}} r_{i j}\right| \psi\right\rangle
$$

is the average of the shortest distance between two electrons with the same spin, one "reference" electron being located at $\mathbf{r}$, and

$$
d_{\sigma \bar{\sigma}}(\mathbf{r})=\left\langle\psi\left|\sum_{i=1}^{N} \delta\left(\mathbf{r}-\mathbf{r}_{i}\right) \min _{j ; \sigma_{i} \neq \sigma_{j}} r_{i j}\right| \psi\right\rangle
$$


is the average of the shortest distance between two electrons with opposite spins, one "reference"electron being located at r. By definition, the EPLF takes its values within the interval $[-1,1]$. It gives a local indicator of electron pairing as follows. In regions of space where electrons are unpaired, the average shortest distance between spin-like and spin-unlike electrons are similar, and the EPLF goes to zero. When antiparallel electrons are paired, EPLF takes positive values and when parallel electrons are paired, EPLF takes negative values. We have used this approach with great success to study the bonding in tetraoxygen ${ }^{43}$ and the complex intermolecular interaction of a lithium atom with a thiophene ring. ${ }^{44}$ Quite recently, an approximation of Eqs. (2) and (3) has been proposed in order to allow the analytical evaluation of the EPLF for the standard forms of wavefunctions employed in computational chemistry without resorting to QMC simulations. ${ }^{45}$ It has been shown that the modified EPLF leads to nearly identical shapes when compared to the original EPLF with the advantage of having much faster calculations while avoiding the presence of statistical noise inherent to any Monte Carlo method. The modified formula can be implemented with HF-, CASSCF-, or DFT-based representations by employing any type of basis set. The new EPLF form is based on the following modified definition of the average of the shortest distances:

$$
\begin{aligned}
& d_{\sigma \sigma}(\mathbf{r}) \\
& =\sqrt{-\frac{1}{\gamma(\mathbf{r})} \ln \left\langle\psi\left|\sum_{i=1}^{N} \delta\left(\mathbf{r}-\mathbf{r}_{i}\right) \sum_{j \neq i ; \sigma_{i}=\sigma_{j}}^{N} e^{-\gamma(\mathbf{r})\left|\mathbf{r}_{i}-\mathbf{r}_{j}\right|^{2}}\right| \psi\right\rangle} \\
& d_{\sigma \bar{\sigma}}(\mathbf{r}) \\
& =\sqrt{-\frac{1}{\gamma(\mathbf{r})} \ln \left\langle\psi\left|\sum_{i=1}^{N} \delta\left(\mathbf{r}-\mathbf{r}_{i}\right) \sum_{j ; \sigma_{i}=\sigma_{j}}^{N} e^{-\gamma(\mathbf{r})\left|\mathbf{r}_{i}-\mathbf{r}_{j}\right|^{2}}\right| \psi\right\rangle}
\end{aligned}
$$

where $\gamma(\mathbf{r})$ is an $\mathbf{r}$-dependent exponent directly related to the local electronic density (see details in Ref. 45). In order to study the bonding pattern of the lowest energy isomer of $\mathrm{Be}_{3}$, we have calculated the EPLF using the most dominant part of our best reference wavefunction, namely, the function issued from the CASSCF $(12,15)$ calculation. The construction of the EPLF used all the determinants (around 30000 ) whose CI coefficients are larger than $10^{-5}$. A discussion of the $3 \mathrm{D}$ plots of the EPLF will be presented below.

\section{RESULTS AND DISCUSSION}

\section{A. Structures of both $\mathrm{Be}_{3}$ isomers}

We start by noting the dramatic effect on the reduction of the Be-Be distances when the $1 s$ core electrons are included in the reference space as active; for instance, the optimal Be-Be distance of the linear isomer goes from 4.16 to 4.09 a.u., while for the triangular structure it is reduced from 4.17 to 4.10 a.u. at the CASSCF $(12,12)+$ AQCC/cc-pV5Z level. Table III presents the optimized geometries for the linear and triangular trimers along with previous results at different levels of theory, most of which kept the $1 s$ core elec-
TABLE III. $\mathrm{Be}_{3}$ optimized AQCC/cc-pV5Z geometries (a.u.) and AQCC/aug-cc-pV5Z energies (a.u.). Binding energies in $\mathrm{kcal} / \mathrm{mol}$. BE(CP) means counterpoise corrected values. Energies are reported using the $\operatorname{CASSCF}(12,13)$ and $\operatorname{CASSCF}(12,14)$ reference spaces (see text). Previous values in parentheses. $\mathrm{BE}$ are calculated using the $\operatorname{CASSCF}(4,5)+\mathrm{AQCC}$ atomic energy; for details on the $\mathrm{CP}$ corrections see the text.

\begin{tabular}{lcc}
\hline \hline & CASSCF(12,13) & $\operatorname{CASSCF}(12,14)$ \\
\hline $\mathrm{D}_{3 \mathrm{~h}}$ & $\mathrm{R}_{\mathrm{e}}=4.101\left(4.20^{\mathrm{b}}, 4.21^{\mathrm{c}}, 4.29^{\mathrm{d}}, 4.22^{\mathrm{e}}, 4.38^{\mathrm{f}}, 4.08^{\mathrm{g}}, 4.20^{\mathrm{h}}\right)$ \\
$\mathrm{E}(\mathrm{CASSCF})$ & -43.825969 & -43.841233 \\
$\mathrm{E}($ AQCC) & -43.984548 & $-43.985563\left(-43.882330^{\mathrm{b}}\right)$ \\
$\mathrm{BE}$ & 28.88 & 29.51 \\
$\mathrm{BE}(\mathrm{CP})$ & 26.67 & $27.29\left(22.5^{\mathrm{b}}, 20.4^{\mathrm{c}}, 17.2^{\mathrm{d}}, 19^{\mathrm{e}}, 13.9^{\mathrm{f}}, 21.2^{\mathrm{h}}\right)$ \\
Linear & \multicolumn{2}{c}{$\mathrm{R}_{\mathrm{e}}=4.088\left(4.220^{\mathrm{a}}, 4.26^{\mathrm{b}}, 4.16^{\mathrm{h}}\right)$} \\
$\mathrm{E}(\mathrm{CASSCF})$ & -43.832138 & -43.838818 \\
$\mathrm{E}($ AQCC $)$ & -43.966717 & $-43.967047\left(-43.874507^{\mathrm{a}},-43.868969^{\mathrm{b}}\right)$ \\
$\mathrm{BE}$ & 17.69 & 17.90 \\
$\mathrm{BE}(\mathrm{CP})$ & 16.10 & $16.32\left(11.4^{\mathrm{a}}, 8.84^{\mathrm{b}}, 12.63^{\mathrm{h}}\right)$ \\
\hline \hline
\end{tabular}

${ }^{\mathrm{a}}$ From Ref. 24, FCI with $[5 s 3 p 2 d]$ ANO basis.

${ }^{\mathrm{b}}$ From Ref. 12, MRCI/[5s $\left.3 p 2 d 1 f\right]$.

${ }^{\mathrm{c}}$ From Ref. $12, \operatorname{CCSD}(\mathrm{T}) /[5 s 3 p 2 d 1 f]$

${ }^{\mathrm{d}}$ From Ref. 23, FCI with [3s2p1d] ANO basis set.

${ }^{\mathrm{e}}$ From Ref. 8, MRCI/[7s4p2d].

${ }^{\mathrm{f}}$ From Ref. $13 \mathrm{MRCI} /[4 s 2 p 1 d]$.

${ }^{\mathrm{g}}$ From Ref. 20 B3LYP/6-311++G(3 $\left.\underline{d f}\right)$.

${ }^{\text {h}}$ From Ref. 27, active space CCSDt/cc-pVTZ.

trons frozen. For the latter case, we note that even though the optimizations were done within the more general $\mathrm{C}_{2 \mathrm{v}}$ group, the optimal structure turned out to be an equilateral triangle ( $\mathrm{D}_{3 \mathrm{~h}}$ point group), as found before using other methods, like MP2, MP4, MRCI, and FCI. We can compare the present equilibrium distances with previous results. Sudhakar et al. ${ }^{15}$ report an equilibrium MP2/6-311+G* bond length of 4.23 a.u. for the $\mathrm{D}_{3 \mathrm{~h}}$ isomer. Using a larger basis set, Harrison and $\mathrm{Handy}^{8}$ obtained a Be-Be distance of 4.22 a.u. through the MRCI/[7 $\underline{s} 4 p 2 d]$ method. Junquera et al. ${ }^{23}$ using FCI with the $[3 s 2 p 1 d]$ ANO basis set obtained an equilibrium distance of 4.29 a.u. for the equilateral isomer. Rendell et al. ${ }^{12}$ reported much shorter bond lengths of 4.22 a.u. $[\operatorname{CCSD}(\mathrm{T})]$ and 4.20 a.u. at the MRCI level, but note that their basis set $(5 s 3 p 2 d 1 f)$ already includes one function. The same authors have stressed that both basis set improvements and electron correlation (coupled-cluster, MRCI) shorten the bond length and increase the binding energy. The shortest Be-Be distance reported for this isomer, 4.08 a.u., was obtained by Beyer et al. ${ }^{20}$ with a DFT-based approach, using the B3LYP/6-311++G(3df) method. Lee et al. ${ }^{11}$ obtained $\mathrm{R}_{\mathrm{e}}$ $=4.199$ a.u. by MRCI with a [5s3p2d1f] basis set and their MRCI calculations used a threshold of 0.025 for the CI coefficient of the reference configurations.

More recently we note the active space CCSDt and MRCI calculations of Ref. 27, performed in bases cc-pVTZ and cc-pVQZ, respectively. The active-space CCSDt calculations were an extension of an earlier study ${ }^{28}$ and reproduce quite well the FCI results of Junquera et al.;3 in particular, they report a Be-Be distance of 4.20 a.u for the equilateral isomer. However, in comparison with our 5-active orbital per atom procedure, we stress that they kept the $1 s$ core orbitals frozen, thus neglecting all the core-core and core-valence correlation effects. 
For the linear isomer, there are far fewer studies; however, we point out the recent work by Vetere et al. ${ }^{24}$ using the FCI method to address linear beryllium chains. For the linear $\mathrm{Be}_{3}$ cluster, they obtained an equilibrium distance of 4.22 a.u. with the $5 s 3 p 2 d$ ANO basis. However, note here that with the $4 s 3 p 1 d$ basis set, the FCI distance is significantly longer (4.28 a.u.), thus revealing the very strong dependence of the equilibrium distance on the basis set quality. The newest highly correlated results come from Klos et al., ${ }^{27}$ where they obtain a Be-Be distance of 4.15 a.u. for the linear isomer at the CCSDt/cc-pVTZ level but, again, neglecting all the core-core and core-valence correlation effects. The present CASSCF $(12,12)+A Q C C / c c-p V 5 Z$ optimized geometry yields $R_{e}=4.088$ a.u. and it was obtained with a significantly larger basis set including much higher l-angular momentum functions up to $3 f 2 g 1 h$. The crucial factor here is that our reference CASSCF wavefunctions were built considering the six $1 s$ core electrons as active, while in the calculations of Refs. 23, 27, and 28 these were kept frozen, i.e., the three lowest MO are doubly occupied in all the configurations of their FCI and CCSDt expansions.

\section{B. Binding energies}

The abundant literature on $\mathrm{Be}_{3}$ shows that the binding energy is extremely dependent upon the type of correlation treatment and the quality of the basis sets employed. Let us first begin with the triangular isomer. Sudhakar and Lammertsma ${ }^{15}$ reported a detailed analysis with the $6-31 \mathrm{G}^{*}$ and $6-31+\mathrm{G}^{*}$ basis sets at various levels of electronic correlation using coupled cluster (CC) and Möller-Plesset theories; their dissociation energies were $31.4 \mathrm{kcal} / \mathrm{mol}$ at MP2/6$31 \mathrm{G}^{*}$ and $18.7 \mathrm{kcal} / \mathrm{mol}$ at MP4/6-31G* which illustrate a large overestimation of correlation effects with MP2, while their $21.6 \mathrm{kcal} / \mathrm{mol}$ value at MP4/6-311+G* shows the dependence on basis set quality. Rendell et al. ${ }^{12}$ report a $\mathrm{D}_{\mathrm{e}}$ of $22.5 \mathrm{kcal} / \mathrm{mol}$ at the MRCI/[5321] level and $20.6 \mathrm{kcal} / \mathrm{mol}$ after correction for harmonic zero-point vibrational energy contributions. They found a much smaller binding energy of $11.3 \mathrm{kcal} / \mathrm{mol}$ at the CCSD level with the same basis set, but a value of $20.4 \mathrm{kcal} / \mathrm{mol}$ when the triple excitations are included at the $\operatorname{CCSD}(\mathrm{T})$ level. Watts et al $^{13}$ reported 16.2, 11.3 , and $13.9 \mathrm{kcal} / \mathrm{mol}$ values using fourth-order many-body perturbation theory (MBPT)(4), CCSD(T), and MRCI respectively, all using a $4 s 2 p 1 d$ ANO basis set. However, their MRCI calculations used the CASSCF reference that arises with the $2 s$ and $2 p$ orbitals as active, keeping the three $1 s$ orbitals frozen, thereby excluding all the core-valence correlation effects. Harrison and $\mathrm{Handy}^{8}$ calculated a MRCI value of $19 \mathrm{kcal} / \mathrm{mol}$ with a $7 s 4 p 2 d$ basis set, but they estimated a true binding energy of $24 \pm 2 \mathrm{kcal} / \mathrm{mol}$ by including extrapolation of higher excitations and basis set extensions. Junquera et al., ${ }^{23}$ through full CI calculations using the $3 s 2 p 1 d$ ANO basis set, reported a value of $17.2 \mathrm{kcal} / \mathrm{mol}$; however, they also kept the three $1 s$ orbitals frozen (doubly occupied) in all the determinants of their FCI wavefunction. The newest estimations of the binding energy for $\mathrm{Be}_{3}$ come from the active space CCSDt/cc-pVTZ calculations of Klos et $a{ }^{27}$ where they report $\mathrm{BE}$ of 21.2 and $12.63 \mathrm{kcal} / \mathrm{mol}$ for the equilateral and linear isomers, respectively; however, we emphasize that they also kept the $1 s$ core orbitals frozen, thereby neglecting the core-core and core-valence correlation effects. Our CASSCF $(12,14)+$ AQCC/AV5Z binding energy is $29.5 \mathrm{kcal} / \mathrm{mol}$ but, as expected, the BSSE is non-negligible (around $10 \%$ of the $\mathrm{BE}$ ) so that when the $\mathrm{CP}$ correction is applied, a value of $27.3 \mathrm{kcal} / \mathrm{mol}$ is obtained. This new benchmark value is larger than all previous estimates, but it remains close to the upper limit of the prediction made by Harrison and Handy ${ }^{8}$ twenty-five years ago concerning the exact binding energy.

For the linear cluster, Rendell et al. ${ }^{12}$ report an MRCI dissociation energy of $8.8 \mathrm{kcal} / \mathrm{mol}$ with the $[5 s 3 p 2 d 1 f]$ basis set. Vetere et $\mathrm{al}^{24}{ }^{24}$ performed systematic FCI calculations using increasingly larger ANO basis sets. They found that the smaller basis sets lead to dissociative potential curves and that only the largest basis sets yield stable linear $\mathrm{Be}_{3}$ clusters. Their FCI/[5s3p2d] dissociation energy is $11.4 \mathrm{kcal} / \mathrm{mol}^{46}$ without taking into account the basis set superposition error, but we recall that their FCI calculations kept the $1 s$ orbitals frozen.

Our CASSCF $(12,14)+A Q C C / A V 5 Z$ dissociation energy is $17.9 \mathrm{kcal} / \mathrm{mol}$ and, even when the $\mathrm{CP}$ correction is applied, it remains slightly larger $(16.3 \mathrm{kcal} / \mathrm{mol})$ than previous estimates.

\section{Analysis of the bonding pattern in the equilateral isomer through the EPLF}

Since the EPLF is a scalar field in ordinary space, there are two easy ways to visualize it. The first one is to plot the points in space where the EPLF takes on a specified value (called the threshold in what follows). Of course the shape and extension of the resulting isosurface will change with varying thresholds. Figures 1 and 2 show the surfaces of the EPLF obtained with two isosurface values. In both isosurfaces, the three $1 s^{2}$ couples of spin-paired electrons appear as spheres centered around each nucleus. However, it is quite interesting to note that the first isosurface (at 0.011 ) also reveals a larger "ring" which completely surrounds the nuclear skeleton and has three radial arms between each couple of Be atoms. If the threshold is slightly increased to 0.0113 , the isosurface varies, the surrounding ring disappears, and the regions between the Be atoms become more dominant.

\section{CONCLUSIONS AND PERSPECTIVES}

In this work, we have reported the results of benchmark type multireference variational CI calculations. The optimal geometries and binding energies for the linear and triangular isomers of the beryllium trimer have been obtained with very large basis sets and considering the six $1 s$ core electrons as active. The calculations have been done using a twostep procedure. First, starting from the best previously reported geometries, we have reoptimized the geometries of both isomers at the AQCC level using very large complete active space SCF references with 12 active electrons in 12 orbitals with the cc-pV5Z basis set. Then, using these optimized 


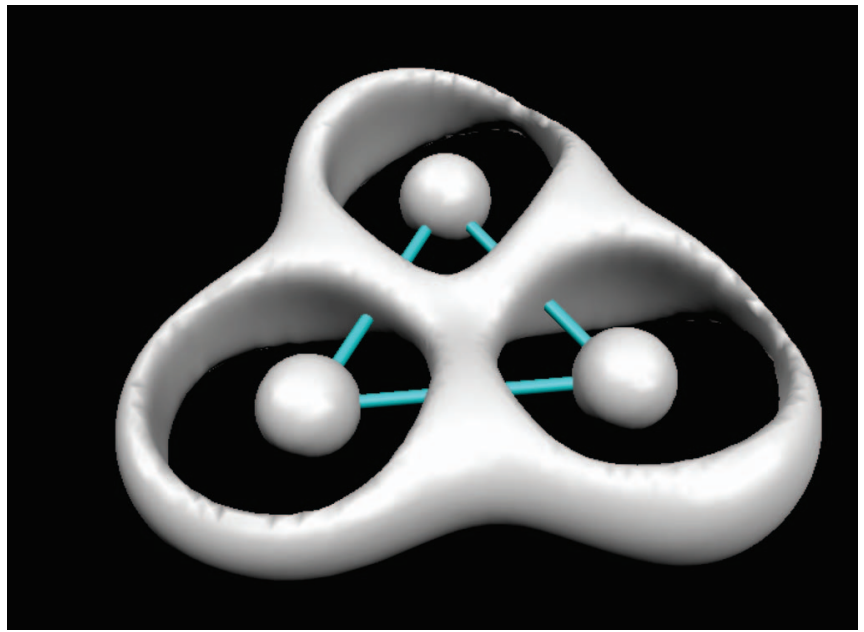

FIG. 1. EPLF of the equilateral $\mathrm{Be}_{3}$ isomer. EPLF calculated with all the determinants in the CASSCF $(12,15)$ wavefunction whose $\mathrm{CI}$ coefficients are larger than $10^{-5}$. Isosurface value of 0.011 .

geometries, we have performed single-point AQCC/aug-ccpV5Z calculations using even larger complete active space SCF references with 12 active electrons in 13 and 14 orbitals. The binding energies were counterpoise $(\mathrm{CP})$ corrected to account for the basis set superposition errors. The present CPcorrected binding energies, 27.3 and $16.3 \mathrm{kcal} / \mathrm{mol}$ for the equilateral and linear isomers, are larger than the previous FCI benchmark values. The equilibrium distances of 4.101 a.u. for the equilateral isomer and 4.088 a.u. for the linear cluster are slightly shorter than the previous best estimates. These effects are mainly due to the inclusion of the six $1 s$ core electrons as active in our AQCC benchmark calculations.

To visualize the nature of the bonding for the $\mathrm{D}_{3 \mathrm{~h}}$ isomer, we have calculated the EPLF function and the plots clearly show the crucial role played by the $2 p$ orbitals lying in the molecular plane.

Since the AQCC method has been shown to yield parallel potential curves to the FCI ones for Be clusters and since

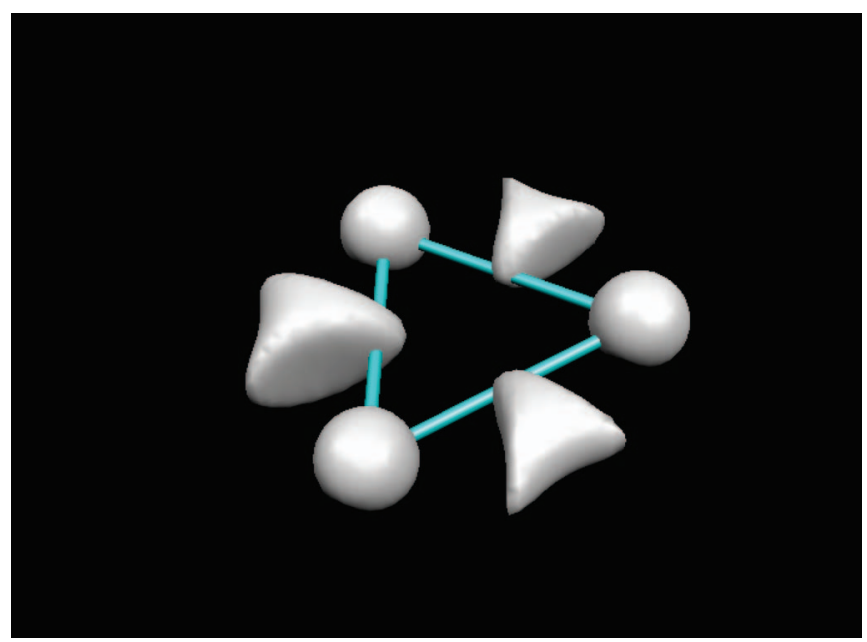

FIG. 2. EPLF of the equilateral $\mathrm{Be}_{3}$ isomer. EPLF calculated with all the determinants in the $\operatorname{CASSCF}(12,15)$ wavefunction whose $\mathrm{CI}$ coefficients are larger than $10^{-5}$. Isosurface value of 0.0113 . we have included the six $1 s$ core electrons as active (which leads to the inclusion of up to $4.7 \times 10^{10} \mathrm{CSFs}$ ), we believe that the geometries and energies presented here are the most accurate to date and, thus, should be used as reference data. The MRCI calculations based on the ideal chemically relevant reference space (namely, all 12 electrons in 15 active orbitals) using the very large AV5Z basis set are still unfortunately too large to be feasible with present-day computational resources. Finally, let us note that work is under way to obtain the best estimate of the binding energy for $\mathrm{Be}_{3}$ through fixed-node diffusion quantum Monte Carlo calculations using the present CASSCF $(12,15)$ reference as trial wavefunction at the optimized geometry with the cc-pV5Z basis set.

\section{ACKNOWLEDGMENTS}

A.R.-S. thanks support from CONACYT Project No. 130931. The present work summarizes the M.Sc. thesis of J.I.A.-E. who acknowledges a CONACYT graduate scholarship. We thank unlimited CPU time from the UAEM supercomputing center and from CALMIP-Université de Toulouse.

${ }^{1}$ W. Kolos, F. Nieves, and O. Novaro, Chem. Phys. Lett. 41, 431 (1976).

${ }^{2}$ L. W. Bruch, O. Novaro, and A. Flores, J. Chem. Phys. 67, 2371 (1977).

${ }^{3}$ B. Liu and A. D. McLean, J. Chem. Phys. 72, 3418 (1980).

${ }^{4}$ R. A. Whiteside, R. Krishnan, J. A. Pople, M.-B. Krogh-Jespersen, P. R. Schleyer, and G. Wenke, J. Comput. Chem. 1, 307 (1980).

${ }^{5}$ E. Blaisten-Barojas and S. N. Khanna, Phys. Rev. Lett. 61, 1477 (1980).

${ }^{6}$ V. E. Bondybey, Chem. Phys. Lett. 109, 436 (1984).

${ }^{7}$ V. E. Bondybey, Science 227, 125 (1985).

${ }^{8}$ R. J. Harrison and N. C. Handy, Chem. Phys. Lett. 123, 321 (1986).

${ }^{9}$ C. Sosa, J. Noga, and R. J. Bartlett, J. Chem. Phys. 88, 5974 (1988).

${ }^{10}$ R. K. Gosavi and O. P. Strausz, Can. J. Chem. 66, 2034 (1988).

${ }^{11}$ T. J. Lee, A. P. Rendell, and P. R. Taylor, J. Chem. Phys. 92, 489 (1990).

${ }^{12}$ A. P. Rendell, T. J. Lee, and P. R. Taylor, J. Chem. Phys. 92, 7050 (1990).

${ }^{13}$ J. D. Watts, I. Cernusak, J. Noga, R. J. Bartlett, C. W. Bauschlicher, Jr., T. J. Lee, A. P. Rendell, and P. R. Taylor, J. Chem. Phys. 93, 8875 (1990).

${ }^{14}$ J. Noga, W. Kutzelnigg, and W. Klopper, Chem. Phys. Lett. 199, 497 (1992).

${ }^{15}$ P. V. Sudhakar and K. Lammertsma, J. Chem. Phys. 99, 7929 (1993).

${ }^{16}$ S. Evangelisti, G. L. Bendazzoli, and L. Gagliardi, Chem. Phys. 185, 47 (1994).

${ }^{17}$ L. Füsti-Molnar and P. G. Szalay, Chem. Phys. Lett. 258, 400 (1996).

${ }^{18}$ S. Evangelisti, G. L. Bendazzoli, R. Ansaloni, F. Durì, and E. Rosi, Phys. Chem. Chem. Phys. 252, 437 (1996).

${ }^{19}$ L. A. Kaledin, A. L. Kaledin, M. C. Heaven, and V. E. Bondybey, J. Mol. Struct.: THEOCHEM 461, 177 (1999).

${ }^{20}$ M. K. Beyer, M. K. Beyer, L. A. Kaledin, A. L. Kaledin, M. C. Heaven, and V. E. Bondybey, Chem. Phys. 262, 15 (2000).

${ }^{21}$ I. Kaplan, S. Roszak, and J. Leszczynski, J. Chem. Phys 113, 6245 (2000).

${ }^{22}$ B. O. Roos and P. O. Widmark, in European Summer School in Quantum Chemistry, Book III (University of Lund, Lund, 2003).

${ }^{23}$ J. M. Junquera, J. Sánchez-Marín, G. L. Bendazzoli, and S. Evangelisti, J. Chem. Phys. 120, 8405 (2004).

${ }^{24}$ V. Vetere, A. Monari, A. Scemama, G. L. Bendazzoli, and S. Evangelisti, J. Chem. Phys. 130, 024301 (2009).

${ }^{25}$ K. Patkowski, R. Podeszwa, and K. Szalewicz, J. Phys. Chem. A 111, 12822 (2007).

${ }^{26}$ M. W. Schmidt, J. Ivanic, and K. Ruedenberg, J. Phys. Chem. A 114, 8687 (2010).

${ }^{27}$ J. Klos, P. S. Zuchowski, L. Rajchel, G. Chalasinski, and M. M. Szczesniak, J. Chem. Phys. 129, 134302 (2008).

${ }^{28}$ K. Kowalski, S. Hirata, M. Wloch, P. Piecuch, and T. L. Windus, J. Chem. Phys. 123, 074319 (2005).

${ }^{29}$ J. M. Merritt, V. E. Bondybey, and M. C. Heaven, Science 324, 1548 (2009) 
${ }^{30}$ C. Filippi and C. J. Umrigar, J. Chem. Phys. 105, 213 (1996).

${ }^{31}$ F. Schautz, H. J. Flad, and M. Dolg, Theor. Chem. Acc. 99, 231 (1998).

${ }^{32}$ S. Zhang and H. Krakauer, Phys. Rev. Lett. 90, 136401 (2003).

${ }^{33}$ M. Casula, C. Attaccalite, and S. Sorella, J. Chem. Phys. 121, 7110 (2004).

${ }^{34}$ J. A. W. Harkless and K. K. Irikura, Int. J. Quantum Chem. 106, 2373 (2006).

35 J. Toulouse and C. J. Umrigar, J. Chem. Phys. 128, 174101 (2008).

${ }^{36}$ A. G. Anderson and W. A. Goddard III, J. Chem. Phys. 132, 164110 (2010).

${ }^{37}$ MOLPRO internal cc-pV5Z Be basis set from T. H. Dunning, Jr., J. Chem. Phys. 90, 1007 (1989).

${ }^{38}$ B. P. Prascher, D. E. Woon, K. A. Peterson, T. H. Dunning, Jr., and A. K. Wilson, Theor. Chem. Acc. 128, 69 (2011).

${ }^{39}$ P. G. Szalay and R. J. Bartlett, Chem. Phys. Lett. 214, 481 (1993).
${ }^{40}$ MOLPRO, a package of ab initio programs designed by H.-J. Werner and P. J. Knowles, version 2006.1, F. R. Manby, M. Schütz, R. Lindh et al.

${ }^{41}$ S. F. Boys and F. Bernardi, Mol. Phys. 19, 553 (1970).

${ }^{42}$ A. Scemama, P. Chaquin, and M. Caffarel, J. Chem. Phys. 121, 1725 (2004).

${ }^{43}$ A. Scemama, M. Caffarel, and A. Ramírez-Solís, J. Phys. Chem. A 113, 9014 (2009).

${ }^{44}$ M. Caffarel, A. Scemama, and A. Ramírez-Solís, Theor. Chem. Acc. 126, (2010).

${ }^{45}$ A. Scemama, M. Caffarel, R. Chaudret, and J. P. Piquemal, J. Chem. Theory Comput. 7, 618 (2011).

${ }^{46}$ This value was obtained using the energy of the cluster reported at 4.25 a.u. (see Table III of Ref. 24), while their optimal Be-Be distance is 4.22 a.u., thus yielding a lower bound value. 